\title{
Integrated analysis of long non-coding RNAs and mRNA expression profiles reveals the potential role of IncRNAs in gastric cancer pathogenesis
}

\author{
XIAO-CONG LIN ${ }^{1}$, YING ZHU ${ }^{2}$, WEN-BIAO CHEN ${ }^{2}$, LIE-WEN LIN ${ }^{2}$, DE-HENG CHEN ${ }^{2}$, JIAN-RONG HUANG ${ }^{2}$, \\ KAI PAN ${ }^{2}$, YAN LIN ${ }^{1}$, BI-TAO WU ${ }^{1}$, YONG DAI ${ }^{2}$ and ZHI-GUANG TU ${ }^{1}$ \\ ${ }^{1}$ Key Laboratory of Laboratory Medical Diagnostics, Ministry of Education, Chongqing Medical University, \\ Chongqing 400016; ${ }^{2}$ The Second Clinical Medical College, Jinan University, \\ Shenzhen People's Hospital, Shenzhen 518020, Guangdong, P.R. China
}

Received February 8, 2014; Accepted April 24, 2014

DOI: $10.3892 /$ ijo.2014.2431

\begin{abstract}
Long non-coding RNAs (lncRNAs) have been shown to play a critical role in cancer biology and are frequently aberrantly expressed. Despite their important role in pathology, little is known mechanistically regarding their role in gastric cancer (GC) pathogenesis. To characterize the role of IncRNAs in GC pathogenesis, 8 paired human GC tissue samples and matched adjacent normal tissue were examined. Large scale expression profiling of lncRNA and mRNA was performed utilizing microarray technology and validated by qPCR. Differentially expressed lncRNAs were subjected to bioinformatic analysis to predict target genes, followed by the integration of differentially expressed mRNA data and GO and network analysis to further characterize potential interactions. In our study, 2,621 lncRNAs and 3,121 mRNAs were identified to be differentially expressed ( $\geq 2$.0-fold change) in GC samples relative to their matched counterparts. IncRNA target prediction revealed the presence of 221 potential lncRNA-mRNA target pairs for the 75 differentially expressed lncRNAs and 60 differentially expressed genes. KEGG pathway analysis showed that these target genes were significantly enriched in
\end{abstract}

Correspondence to: Professor Zhi-Guang Tu, Key Laboratory of Laboratory Medical Diagnostics, Ministry of Education, Chongqing Medical University, 1 Yixueyuan Road, Yuzhong District, Chongqing 400016, P.R. China

E-mail: tuzhiguang@126.com

\begin{abstract}
Abbreviations: lncRNAs, long non-coding RNAs; GC, gastric cancer; GO, Gene Ontology; ORF, open reading frames; GEO, Gene Expression Omnibus; KEGG pathway, Kyoto encydopedia of gene and genomes; ds-cDNA, double-stranded cDNA; ncRNAs, non-coding RNAs; miRNA, microRNA; JAK-STAT signaling pathway, Janus kinase and signal transducer and activator of transcription signaling pathway
\end{abstract}

Key words: gastric cancer, long non-coding RNAs, mRNA, microarray, pathway, network
7 different pathways, of which the p53 signaling pathway was the most significant and has been previously implicated in GC pathogenesis. Construction of a lncRNA-mRNA correlation network revealed 10 differentially expressed lncRNAs potentially regulating the p53 signaling pathway. Overall, this is the first study perform global expression profiling of lncRNAs and mRNAs relating to GC. These results may provide important information for further insights into the pathogenesis of GC and provide potential targets for future therapeutics.

\section{Introduction}

Gastric cancer (GC) is currently the fourth most common malignancy and the second leading cause of cancer-related deaths worldwide $(1,2)$. It is estimated that more than 900,000 new cases were diagnosed and over 700,000 people died from GC in $2011(3,4)$. Due to the absence of specific symptoms, patients are usually not diagnosed until reaching an advanced stage or metastasis (5). Surgical resection is the most effective treatment modality for GC, yet up to half of the patients experience recurrence after curative surgery with a 5-year survival rate of only 20-30\% (1,6). Despite efforts of multiple fields, current interventional strategies have shown little success in increasing the disease-free survival rate of GC patients due to a limited understanding of the disease causing mechanisms (6). Therefore, it is imperative to conduct an in-depth study characterizing GC pathogenesis to identify novel molecular makers and therapeutic targets.

Protein-coding sequences cover only a small fraction ( $2 \%)$ of the human genome, with up to $70 \%$ of the genome transcribed as ncRNAs $(7,8)$. Over the past decade, much attention has focused on miRNA, a class of short ncRNAs ranging in length from 21-25 nucleotides, and their involvement in proliferation, development, differentiation, apoptosis, tumorigenesis and other biological processes (9-11). Recently, a large number of lncRNAs have been found in mouse, fly and human systems (12). Unlike miRNAs, lncRNAs code transcripts longer than 200 nucleotide, with mRNA-like properties including 5 ' capping, splicing and polyadenylation, yet lack a significant ORF $(8,13)$. LncRNAs can regulate gene expression through 
cis- or trans-acting pathways (14), and have been shown to play important roles in a broad range of biological processes such as genomic imprinting, nuclear-cytoplasmic trafficking, dosage compensation, immune responses, developmental patterning and differentiation (15-17). Although thousands of lncRNAs have been identified, the exact function of most lncRNAs remains unknown requiring characterization to reveal the mechanisms by which they regulate biological processes $(12,18)$. However, unlike protein-coding genes whose sequence motifs are usually indicative of their function, the sequences of most lncRNAs are poorly conserved rendering a direct functionality prediction difficult (14). Moreover, evidence indicates that dysregulation of lncRNAs is associated with a wide range of human diseases, possibly through the regulation or interactions of lncRNAs with protein-coding genes $(14,18,19)$. These interactions could ultimately contribute to tumor pathogenesis, making an understanding of these molecular mechanisms imperative. Recently, Cao et al (20) identified a set of differentially expressed lncRNAs in GC by analyzing publicly available human exon array data sets from the GEO, yet the mechanistic properties of this dysregulation remains unknown. In addition, integrated analysis correlating changes in the expression patterns of IncRNAs and mRNAs, as it relates to GC pathogenesis, requires examination.

In this study, we analyzed lncRNA and mRNA expression profiles in samples of GC tissues, in conjunction with paired adjacent normal tissues, utilizing microarray technology. Differentially expressed lncRNAs were subjected to bioinformatic analysis for target gene prediction and findings integrated with differentially expressed mRNA data to increase predictive accuracy. GO and pathway analysis was performed to determine associated functions of the predicted targets, ultimately leading to the construction of a lncRNA-mRNA correlation network. Our results demonstrate that the altered expression levels of lncRNAs and mRNA may have implications in GC pathogenesis, and that integrative analysis of lncRNAs and mRNA may provide a new foundation for diagnosing and treating GC.

\section{Materials and methods}

Patient samples and ethics statement. Eight primary gastric adenocarcinoma samples with paired adjacent normal tissue ( $\geq 5 \mathrm{~cm}$ away from the tumor margin) samples were collected at Shenzhen People's Hospital (Shenzhen, China) following gastrectomy or partial gastrectomy. The diagnosis of gastric adenocarcinoma was determined by histopathological examination for the exclusion of precancer lesions such as gastric epithelial dysplasia and intestinal metaplasia. The patient donors did not received chemotherapy or radiotherapy prior to surgical resection and the participant rights were explained prior to consent form signing. All the clinical characteristics of the eight patients with gastric cancer are shown in Table I. This investigation was carried out according to the Helsinki Declaration guidelines on ethical principles for medical research involving human subjects and approved by the ethics committee of the Shenzhen People's Hospital. Written informed consent was obtained from all participants. All tissue samples were snap-frozen and stored in liquid nitrogen until needed for further testing.
RNA extraction. Frozen tissue samples were homogenized by an electric tissue homogenizer, total RNA extracted using TRIzol reagent (Invitrogen, Carlsbad, CA, USA) and a RNeasy kit (Qiagen, Hilden, Germany) according to the manufacturer's protocol, to include a DNase digestion step. RNA quantification and purity was assessed using a NanoDrop ND-1000 spectrophotometer measuring absorbance ratios of $\mathrm{A}_{260} / \mathrm{A}_{280}$ and $\mathrm{A}_{260} / \mathrm{A}_{230}$, with RNA integrity evaluated by standard denaturing agarose gel electrophoresis.

Analysis of lncRNA and mRNA expression profiles. IncRNA and mRNA microarray. The Human 12x135k lncRNA expression microarray (Roche NimbleGen, Inc.) provide global profiling of long transcripts, containing 22,192 lncRNAs and 20,447 protein-coding mRNAs in human genome, each transcript is accurately identified by specific exon or splice junction probes. To improve statistical confidence, each transcript was targeted with 1-5 strategic probes, with ncRNAs shorter than 200 bp or highly similar sequences excluded. To ensure hybridization quality, negative probes and probes for reference genes were printed multiple times. Human lncRNAs and mRNAs were collected from databases such as UCSC, RNAdb, NCBI RefSeq, UCRs and related microarray detection literature, with both represented on a single array to provide consistent hybridization.

RNA labeling and array hybridization. After completing the RNA quality assessment, $5 \mu \mathrm{g}$ of total RNA served as a template to synthesize ds-cDNA using a cDNA synthesis kit (Superscript Double-Stranded cDNA Synthesis kit, Invitrogen). After incubation with $4 \mu \mathrm{g}$ RNase A for $10 \mathrm{~min}$ at $37^{\circ} \mathrm{C}$, ds-cDNA was cleaned and labeled with $\mathrm{Cy} 3$ overnight using a One-Color DNA labeling kit (Roche NimbleGen, Inc.) per the manufacturer's protocol. Cy3 labeled ds-cDNA in hybridization buffer (NimbleGen hybridization component A) was hybridized for $16-20 \mathrm{~h}$ at $42^{\circ} \mathrm{C}$ in a hybridization chamber (Hybridization System-NimbleGen Systems, Inc., Madison, WI, USA). Following hybridization, slides were washed and scanned on a GenePix 4000B microarray scanner (Axon Instruments, Union City, CA, USA).

Data analysis. Scanned microarray image files (TIFF format) were analyzed using NimbleScan software (version 2.5, Roche NimbleGen, Inc.) to extract raw data as pair files for grid alignment and normalization. Spot intensities were obtained by subtracting local background intensity from the total intensity, and intensities were normalization via quantile normalization and Robust Multichip Average (RMA) (NimbleScan software). The probe level ('_norm_RMA.pair) files and mRNA level (*_RMA.calls) files produced following normalization were further analyzed via Agilent GeneSpring Software (version 11.0, Agilent) for further normalization adjustments, with differentially expressed 1 ncRNAs and mRNAs exhibiting a fold change $\geq 2$-fold.

Quantitative PCR. Following total RNA extraction, samples were reverse transcribed to generate cDNA with appropriate primers. qRT-PCR was carried out in triplicate with a total reaction volume of $10 \mu \mathrm{l}\left(5 \mu \mathrm{l}\right.$ of $2 \mathrm{X} \mathrm{\textrm {RT } ^ { 2 }}$ real-time SYBR Green PCR Master Mix (SuperArray Bioscience, Frederick, 
Table I. Clinical characteristics of the eight patients with gastric cancer.

\section{Characteristic}

Gastric cancer patients

\begin{tabular}{ll}
\hline Age (mean \pm SD), years & $58.75 \pm 11.80$ \\
Sex (no.) & 6 \\
Male & 2 \\
Female & \\
Tumor location & 2 \\
Upper third & 1 \\
Middle third & 5 \\
Lower third & \\
Histological type (no.) & 8 \\
Adenocarcinoma & \\
Differentiation of cancer tissue & 1 \\
High differentiation & 5 \\
Moderate differentiation & 2 \\
Poor differentiation & \\
Lauren classification & \\
Intestinal type & 6 \\
Diffuse type & 2 \\
TNM stage & \\
Stage I & \\
Stage II & \\
Stage III & \\
Stage IV & \\
\hline
\end{tabular}

MD, USA), $0.5 \mu \mathrm{l}$ of PCR forward primer $(10 \mu \mathrm{M}), 0.5 \mu \mathrm{l}$ of PCR reverse primer $(10 \mu \mathrm{M}), 2 \mu \mathrm{l}$ of double-distilled water and $2 \mu \mathrm{l}$ of cDNA) and incubated $\left(95^{\circ} \mathrm{C}\right.$ for $10 \mathrm{~min}$, then 40 cycles at $95^{\circ} \mathrm{C}$ for $10 \mathrm{sec}, 60^{\circ} \mathrm{C}$ for $60 \mathrm{sec}$ ). After PCR amplification, melt curve analysis was performed to confirm reaction specificity; with Human U6 snRNA (for lncRNAs) and 18S rRNA (for mRNA) used as internal controls. Expression fold changes were calculated via the $2^{-\Delta \Delta \mathrm{Ct}}$ method (21). Differences in gene expression levels between GC samples and their paired adjacent tissue sample were analyzed using Student's t-test, with a p-value $<0.05$ considered statistically significant. All primers used in qRT-PCR and cDNA synthesis are shown in Tables II and III.

Association analysis of the different expression of lncRNAs and $m R N A s$

Target genes prediction for different expression lncRNAs. To unveil potential associations of lncRNAs with mRNAs, differentially expressed lncRNAs (fold change, $\geq 3.0$ ) were subjected to bioinformatic analysis for target gene prediction. Two independent algorithms were applied to predict possible target genes for both cis- or trans-acting lncRNAs independently.

In the cis-acting analysis, lncRNAs and their potential paired target genes were obtained and visualized using the UCSC genome browser and genome annotation. The algorithms included an ORF-Predictor and BLASTP pipeline $(\mathrm{e}<1 \mathrm{E}-5)$ to identify lncRNA genes in the human genome and searched for their nearest known protein-coding gene according to distance stratification. The genes located within 10 and 10-300 kb away from lncRNAs were considered as cis10k and cis300k target genes, respectively $(22,23)$. Generally, an equidirectional transcriptive target gene is for promoting expression in the promoter region, otherwise it is for suppression. In some conditions, a reversed direction is possible to promote expression in the 3'-UTR region. The predictive algorithms for the cis-acting analysis were performed as previously described (24-26).

For the trans-acting analysis, a RNAplex program (http:// www.bioinf.uni-leipzig.de/Software/RNAplex/) was used to identify possible target genes for IncRNAs. RNAplex was especially designed to quickly localize possible hybridization sites for a query RNA in large RNA databases by applying a simpler energy model in the first screening phase followed by a full energy model to refine potential binding sites $(27,28)$. First, we performed BLAST $(\mathrm{e}<1 \mathrm{E}-5)$ between $\operatorname{lncRNA}$ and the known protein-coding genes followed by RNAplex software to further screening the trans-acting target genes (parameters were set as -e -20). The RNAplex program for trans-acting analysis was performed as previously described $(22,25-28)$.

Combination of differentially expressed mRNAs with target prediction. To increase the accuracy of target prediction, differentially expressed $\mathrm{mRNAs}$ (fold change, $\geq 3.0$ ) were integrated with the predicted potential of lncRNA targets.

Gene Ontology (GO) analysis and pathway analysis. GO (www.geneontology.org) is a functional analysis utilizing GO categories to describe genes and gene product attributes including molecular function, cellular components and biological processes. To understand the potential roles of differentially expressed lncRNAs, we applied GO categories to analyze the biological functions for the correlated lncRNAs/ gene targets. Additionally, we used the KEGG database (http:// www.genome.jp/kegg/) to identify significant pathways for predicted target genes. GO term enrichment and the biological pathways utilized significant p-values (recommended p-value $<0.05$ ) relating to the target genes of differentially expressed lncRNAs.

LncRNA-mRNA correlation network. Superimposing lncRNA target predictions with differentially expressed lncRNAs and mRNA profiles in Cytoscape (http://www.cytoscape. org) enabled the construction of a lncRNA-mRNA correlation network. As shown in Fig. 5, in this network, circular nodes represent mRNA, triangular nodes represent lncRNA, while red nodes represent an upregulation and green nodes a downregulation relative to matched normal tissue. Direct connections were drawn between IncRNAs and mRNAs using either an orange line (cis-interaction within $10 \mathrm{~kb}$ ), sapphire line (cis-interaction within $10-300 \mathrm{~kb}$ ) or grey line (transinteraction).

Statistical analysis. Statistical analyses were accomplished using SPSS software (version 13.0, SPSS Inc.), with all data expressed as means \pm standard deviation (SD). The Student's $\mathrm{t}$-test was used for evaluating the statistical significance of 
Table II. Primers used for cDNA synthesis and real-time quantitative PCR of lncRNAs.

\begin{tabular}{lllr}
\hline lncRNAs & \multicolumn{1}{c}{ Sense primer for qRT-PCR (5'-3') } & \multicolumn{1}{c}{$\begin{array}{c}\text { Antisense primer for qRT-PCR and } \\
\text { cDNA synthesis (5'-3') }\end{array}$} & Product (bp) \\
\hline uc003iqu & AGTCCCGAATCCCAAGACACT & AAGCTCCATGAACCACCACC & 122 \\
uc003tfx & TCAAGCAATTCTCCTGCCTCA & CACGCCTGTAATCCTAGCACTTT & 189 \\
AK022971 & ATCCCGATTGTTCCCTTTAGTC & CTTTGGTACATGCACGGTTTCT & 250 \\
uc.341 & ACAGCAAAGAGCGGAAGGAA & TCGCGTCAAATACATATTGAACAG & 96 \\
HIV1230 & TTCTTCCCTTTCTACTCACCTTTG & TTCCACCTTCTGCCCTACTTG & 303 \\
BC011663 & AGGTCTGCGTCTGGGAAGG & AGCTCGGCGAAGAGGTGA & 143 \\
AK057054 & CTGTGCTGGCTCCTCTACCTG & TGGGACCTCCTGCCTCTACTT & 99 \\
M14574 & CTGGGCATGTGGAGACAGAG & CAGCCTAAGGGTGGGAAAAT & 89 \\
U6 snRNA & CTCGCTTCGGCAGCACA & AACGCTTCACGAATTTGCGT & 94
\end{tabular}

Table III. Primers used for real-time quantitative PCR of mRNAs.

\begin{tabular}{lllc}
\hline mRNAs & Sense primer (5'-3') & Antisense primer (5'-3') & Product (bp) \\
\hline CCAR1 & TGGATGGACCAGACCCAGAA & CAGGGCGATGGTAGCGAAT & 138 \\
HOXC10 & GAACATCTGGAATCGCCTCA & TCTCCTCTTTCGCTTCGTTATC & 205 \\
RRM2 & GAGAGTAGGCGAGTATCAGAGGA & CAGCCAAGTAAGGGCACATC & 109 \\
DSCR1 & GGACCTGCTCCTTCCTGCTT & GGTTTTCCTTCGATGGCAAA & 249 \\
ALAS1 & AAACAGCCGAGTGCCAAAGTA & GGCATGACTCCATCCCGAT & 261 \\
NPTXR & CCTCGCTTTGGTCATTTGCT & TGAACCTTGCCCTGGACTCT & 184 \\
RBMS2 & TCTCCACCCGTATCCTTCG & CATGACGCCCATGTCTGC & 257 \\
TRIM74 & TCCACTGGTTCCTCCATTCA & AGGCTGGTCTCGAACTCCC & 281 \\
18 srRNA & CCTGGATACCGCAGCTAGGA & GCGGCGCAATACGAATGCCCC & 112 \\
\hline
\end{tabular}

difference in the means between groups with a stringency of $\mathrm{p}<0.05$.

\section{Results}

Aberrant lncRNA and mRNA expression in GC. To investigate the possible biological functions of lncRNAs in GC, we analyzed lncRNA and mRNA expression profiles in GC samples and their matched adjacent normal tissue via microarray technology. Among the 22,192 lncRNA transcripts examined, we found 2,621 (11.8\%) lncRNAs were differentially expressed (fold change, $\geq 2.0$ ) in GC samples relative to their matched counterparts (Fig. 1A and C), with 1,215 being upregulated, while 1,406 were downregulated. Uc010lho (fold change, 39.068) was the most significantly upregulated lncRNA, while HIV1213 (fold change, 56.240) was the most significantly downregulated lncRNA.

The mRNA expression profiling data showed $15.3 \%$ $(3,121 / 20,447)$ of mRNAs to be differentially expressed (fold change, $\geq 2.0$ ) in GC samples relative to their matched counterparts (Fig. 1B and D), with 1,523 upregulated, while 1,598 were downregulated. Among these mRNAs, MUC2 (fold change, 162.24048) showed the highest degree of upregulation, while WDR20 (fold change, 29.62003) was the most downregulated protein-coding gene.
Real-time quantitative PCR validation. To validate the microarray results, 8 differentially expressed lncRNAs and 8 differentially expressed mRNAs were randomly selected and analyzed via qRT-PCR. For the lncRNAs, the results demonstrated that uc003iqu, uc003tfx, AK022971 and uc.341 were upregulated and that HIV1230, BC011663, AK057054 and M14574 were downregulated in the GC tissues relative to their matched counterparts (all p<0.05, Fig. 2A). For the mRNAs, the expression of CCAR1, HOXC10, RRM2, DSCR1, ALAS1, NPTXR, RBMS2 and TRIM74 showed statistically significant differences between matched samples $(\mathrm{p}<0.05$ for each mRNAs, Fig. 2B), with these results being consistent with the microarray data.

Integrating differentially expressed $m R N A$ s into the predicted targets of differentially expressed lncRNAs. Increasing evidence has confirmed that lncRNAs serve an important role by regulating the expression of protein-coding genes. Therefore, we applied bioinformatic analysis to aid in target gene prediction to explore the potential correlation between lncRNA and mRNA expression profiles. First, we used bioinformatic algorithms to predict possible target genes for lncRNAs (fold change, $\geq 3.0$ ). Second, differentially expressed mRNAs (fold change, $\geq 3.0$ ) were integrated with predicted lncRNA targets. The result showed that 60 differentially 


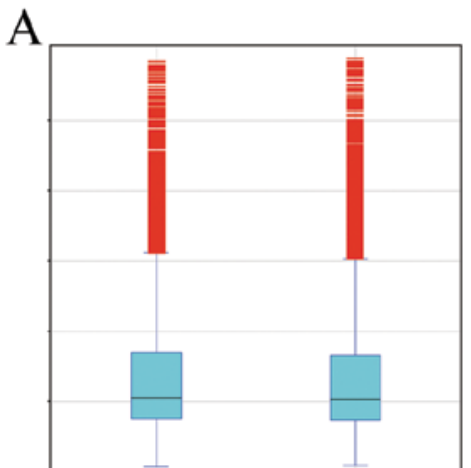

Adjacent normal tissue GC

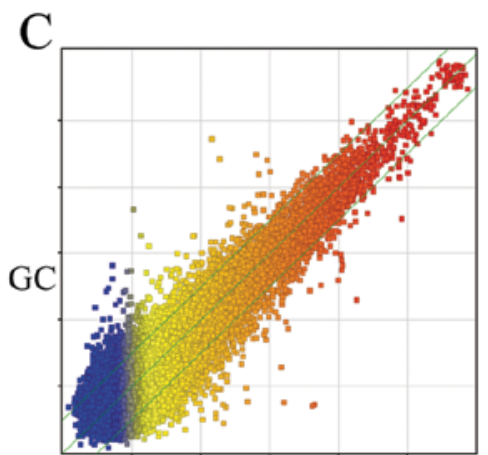

Adjacent normal tissue

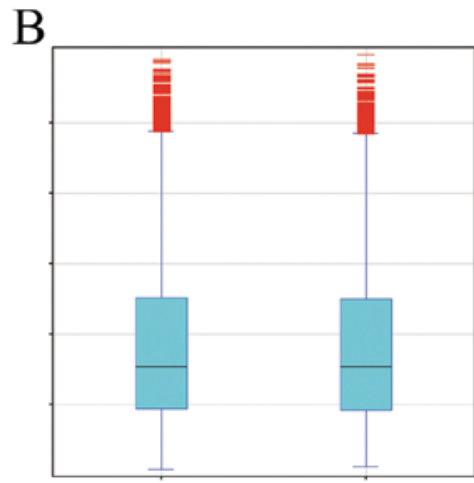

Adjacent normal tissue GC

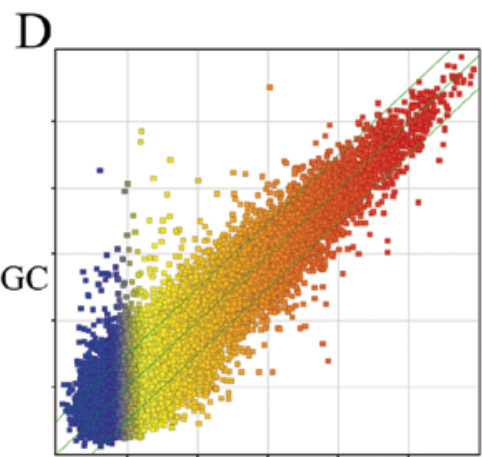

Adjacent normal tissue

Figure 1. IncRNA and mRNA profile comparison between the GC sample and the paired adjacent normal tissue sample. The box plot displays the distribution of a dataset in the lncRNA (A) and mRNA (B) profiles. After normalization, the distributions of $\log _{2}$-ratios among the tested samples are quite similar. The scatter-plot is used for evaluating the lncRNA (C) and mRNA (D) expression variation between GC samples and paired adjacent normal tissue samples. The values of $x$ - and y-axes in the scatter-plot are averaged normalized values for each group $\left(\log _{2}\right.$ scaled). The green lines represent fold change lines (the value of default fold change given is 2.0). The lncRNAs and mRNAs above the top green line and below the bottom green line indicate a $>2$-fold change of 1 ncRNAs and mRNAs between pairs.

A

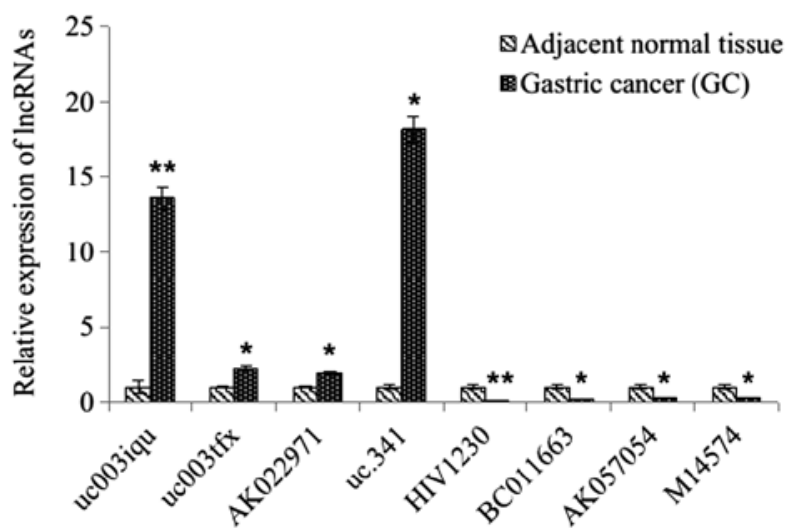

B

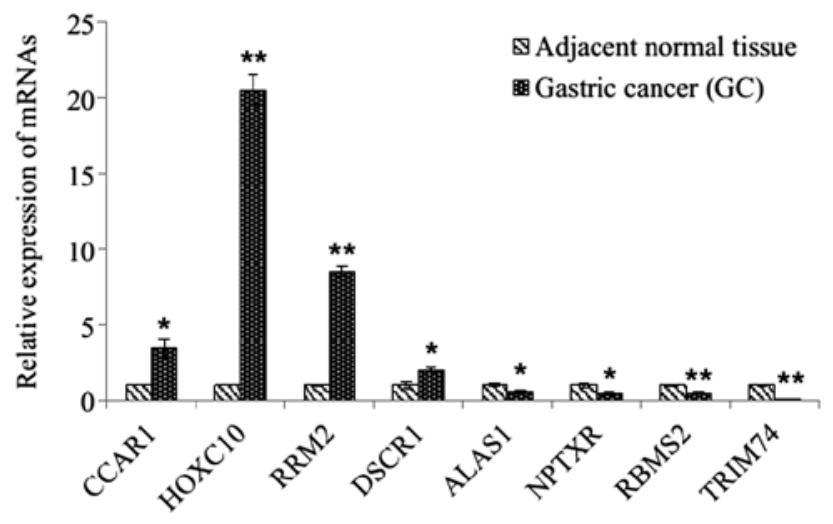

Figure 2. Validation of microarray data by qRT-PCR. The relative expression level of each lncRNA (A) was normalized to U6 snRNA, and each mRNA (B) was normalized to $18 \mathrm{~S}$ rRNA. Data displayed in the histograms are expressed as means $\pm \mathrm{SD},{ }^{*} \mathrm{p}<0.05,{ }^{* *} \mathrm{p}<0.01$ comparing GC samples with adjacent matched normal samples (Student's t-test).

expressed protein-coding genes were significantly associated with each of the 75 differentially expressed lncRNAs to generate 221 lncRNA-mRNA target pairs. Among them, 119 pairs were differentially expressed unidirectionally (up or down), and 102 pairs were differentially expressed bidirectionally. Within our data sets, we found that $3.6 \%(8 / 221)$ of
lncRNA-mRNA target pairs interacted in a cis-manner, while $96.4 \%(213 / 221)$ pairs interacted in a trans-manner.

Ontological and pathway analysis of target genes for differentially expressed lncRNAs. To investigate whether the differentially expressed lncRNAs correlate with functional 


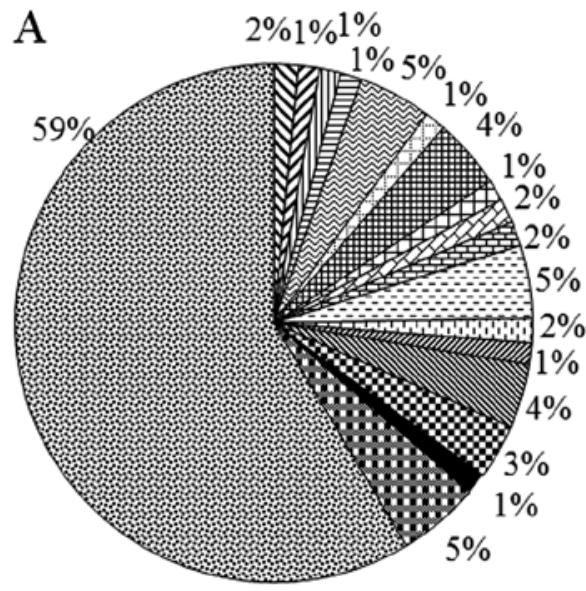

\begin{tabular}{|c|}
\hline$\Delta$ Wound healing \\
\hline m Hemostasis \\
\hline ๑ell communication \\
\hline 田 Signaling \\
\hline$\otimes$ Cell adhesion \\
\hline$\square$ Multicellular organismal process \\
\hline Cell-cell signaling \\
\hline Signal transduction \\
\hline - Response to stimulus \\
\hline
\end{tabular}

Q Blood coagulation

घCoagulation

$\square$ Regulation of body fluid levels

ه Cell activation

田Biological adhesion

$\square$ Response to wounding

$\mathbb{\$}$ Cellular response to stimulus

- Regulation of cell proliferation

잉 Other biological process

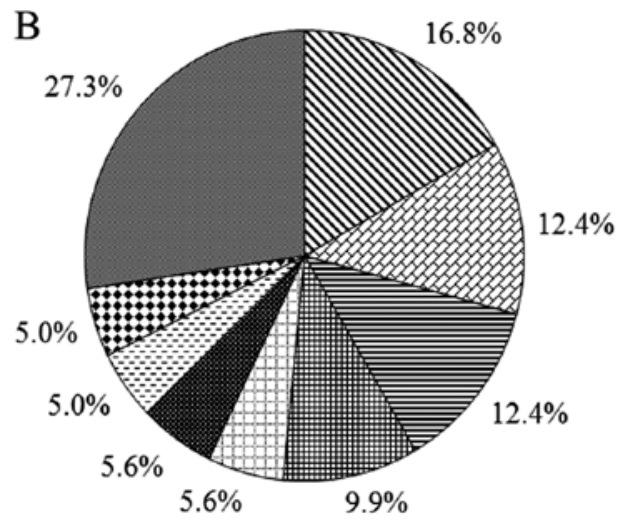

\author{
ब Membrane part \\ ๑ Plasma membrane \\ 目 Cell periphery \\ ⿴囗十 Plasma membrane part \\ $\rightarrow$ Integral to plasma membrane \\ Intrinsic to plasma membrane \\ E Extracellular region part \\ G Cell fraction
}

๑ Other cellular component

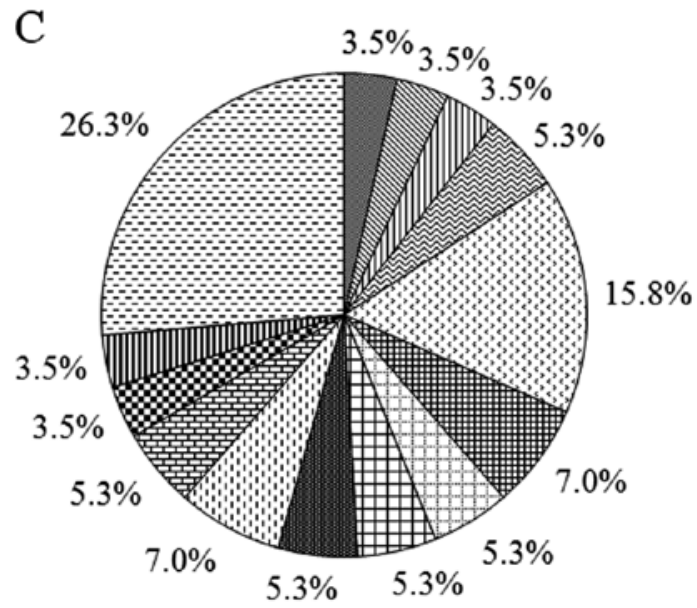

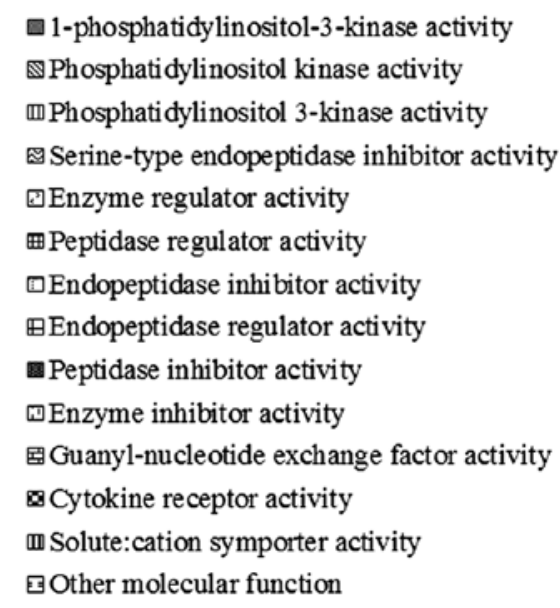

Figure 3. Functional classification of lncRNA target genes based on Gene Ontology (GO). GO analysis was performed to determine the enrichment of lncRNA target genes in biological processes (A), cellular components (B) and molecular functions (C) with significant p-values (recommended $\mathrm{p}<0.05)$.

groupings, GO categories were used to analyze biological process, cellular components and molecular functioning for the correlated targets of specific lncRNAs (Fig. 3). In the GO biological processes classification, most of the lncRNA targets were involved in response to stimuli, cell communication and multicellular organismal processes. The majority of target genes related to cellular components were located in membrane components, the plasma membrane and cell periphery. The GO molecular function classification showed a large proportion of target genes associated with enzyme regulatory activity, peptidase regulatory activity and enzyme inhibitor activity.
Additionally, these target genes were significantly enriched in 7 different pathways (Table IV), of which the p53 pathway $(29,30)$ was the most significant (Table IV and Fig. 4) followed by apoptosis (31) and Jak-STAT signaling pathways $(32,33)$ which have all been previously implicated in GC.

Construction of lncRNA-mRNA correlation network. After merging lncRNA target predictions with lncRNA and mRNA expression profiles, the lncRNA-mRNA correlation network was constructed to include differentially expressed lncRNAs and their target genes (Fig. 5). The correlation network 


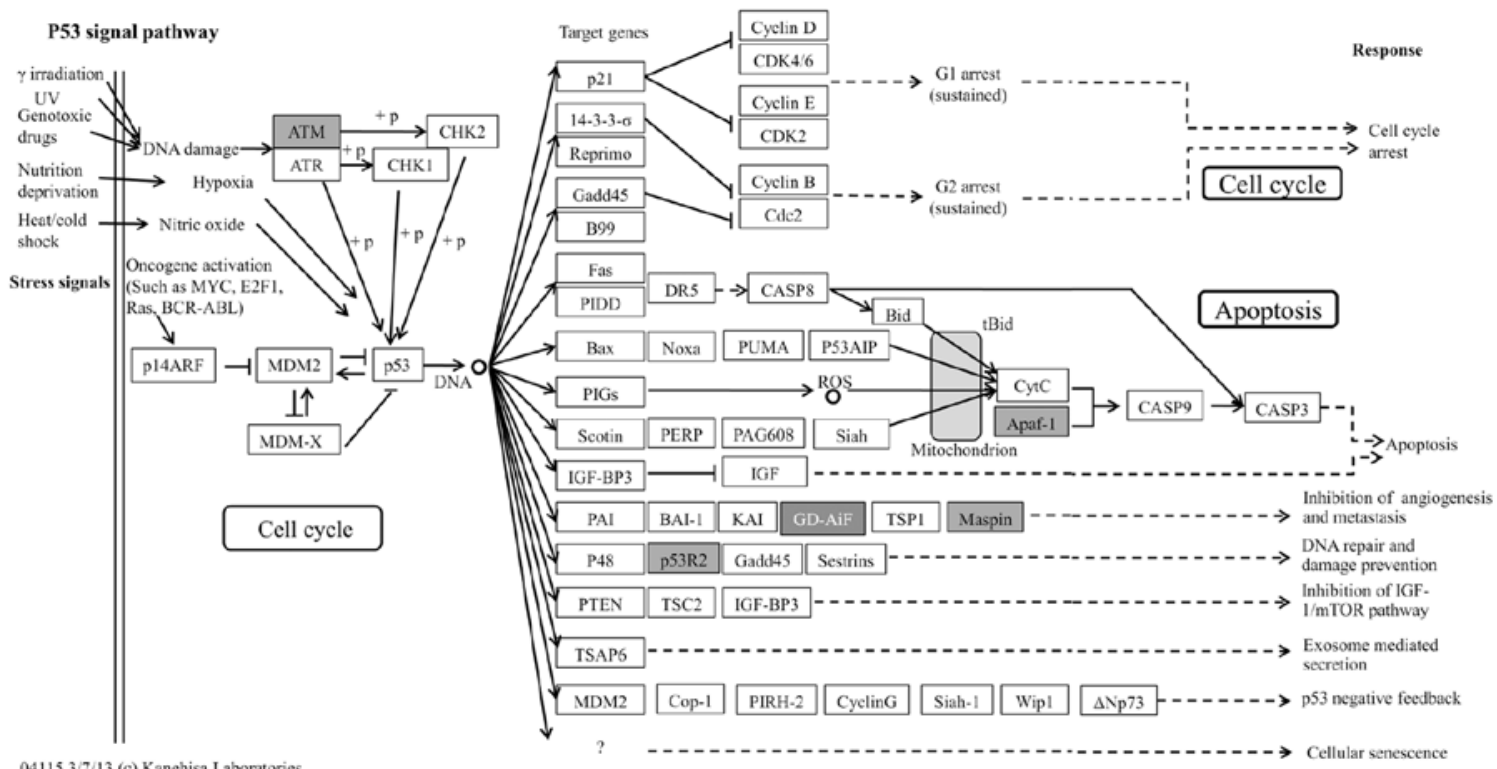

Figure 4. The p53 signaling pathway as it relates to lncRNA target genes. The KEGG database enabled identification of significant pathways (recommended p-value $<0.05$ ) for target genes of lncRNA, with the p53 pathway being the most significantly associated with an outcome of gastric cancer.

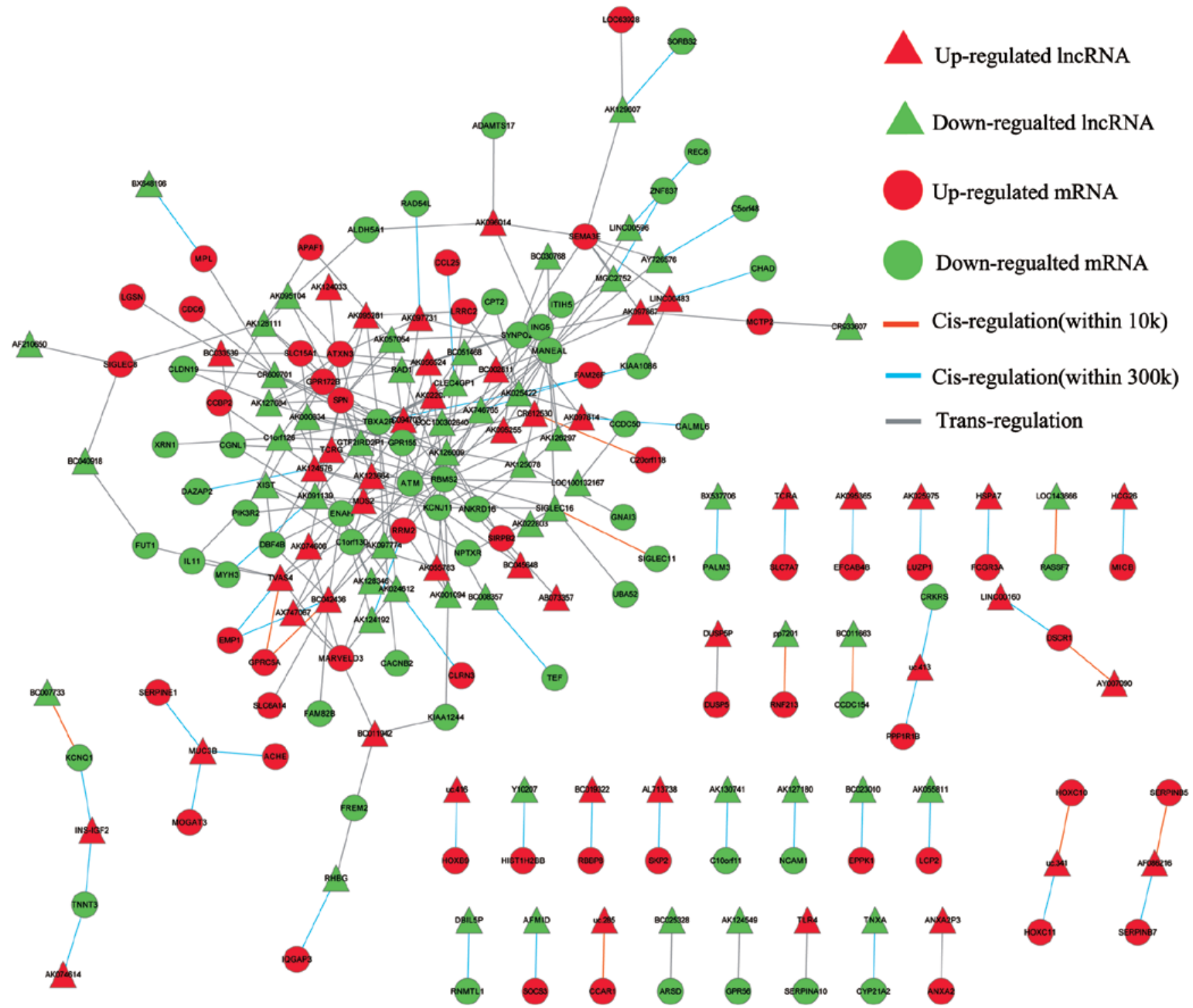

Figure 5. lncRNA-mRNA correlation network. 


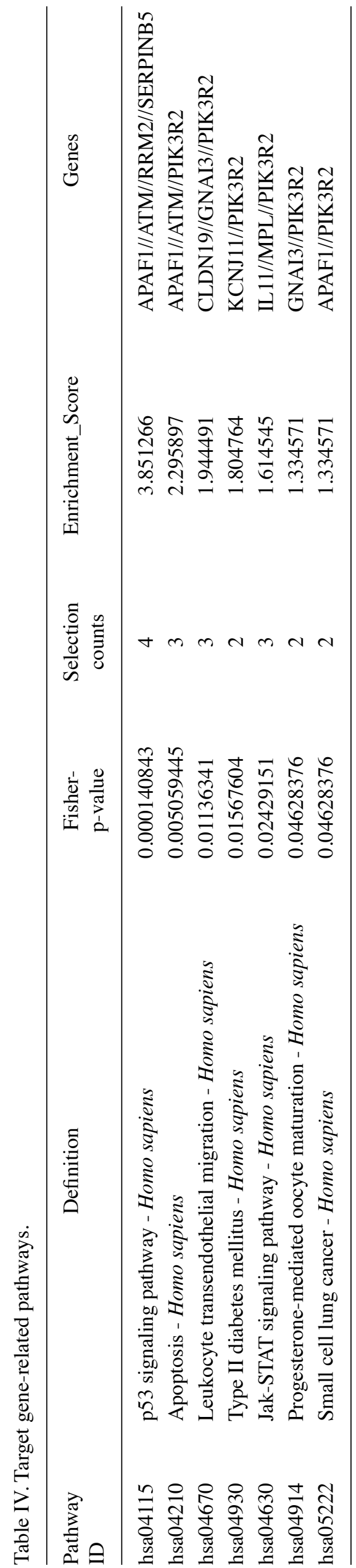

contained 135 nodes and 221 connections between the 75 lncRNAs and the 60 mRNAs. Further analysis of this network indicated that ataxia telangiectasia mutated (ATM) was a predicted target of six lncRNAs (AK001094, AK057054, BC042436, uc003iqu.1, AK022971 and uc003tfx.1), ribonucleotide reductase $\mathrm{M} 2$ polypeptide (RRM2) a predicted target of three IncRNAs (AK091139, AK001094 and uc003dwf.3) and serpin peptidase inhibitor, clade B (ovalbumin), member 5 (SERPINB5) a predicted target of AF086216. These target genes participate in the p53 signaling pathway, and have been previously implicated in the development of GC (34-36). Furthermore, the result also showed that one lncRNA could target up to 5 coding genes and that one coding gene was predicted to be a target of up to 22 lncRNAs in this network.

\section{Discussion}

Gastric cancer is one of the most common and lethal malignancies in humans and comprises $8 \%$ of all cancer cases and $10 \%$ of cancer mortalities worldwide (3). Despite extensive efforts to elucidate the genetic and epigenetic mechanisms involved in disease development and progression, the pathogenesis of GC remains poorly understood to date (37).

Numerous studies in recent decades have demonstrated the importance of lncRNA in cancer pathogenesis, with aberrant expression frequently linked to different kinds of human cancers. Certain lncRNAs, behave like oncogenes or tumor-suppressors displaying an important function in cancer initiation, progression, metastasis and recurrence $(38,39)$. lncRNAs appear to serve a regulatory function by controlling gene expression, making the understanding of their dysregulation and how it relates to cancer pathology essential (19). Hence, constructing lncRNA expression patterns in cancer tissues and isolating potential target molecules is required to enhance cancer diagnostics and therapeutics (38). Recently, a study indicated that the lncRNA H19 was upregulated in GC cells/tissues and that partial inactivation of p53 was linked to the H19 promotion of cellular proliferation and inhibition of apoptosis to display oncogenic functioning (40). However, the functional properties of the vast majority of lncRNAs relating to GC pathogenesis are still unknown. Therefore, we analyzed IncRNA and mRNA expression profiles in GC tissue samples relative to matched normal samples to reveal the potential role of lncRNAs during pathogenesis (Fig. 1). Microarray data revealed $2621 \mathrm{lncRNAs}$ and $3121 \mathrm{mRNAs}$ to be significantly differentially expressed (fold change, $\geq 2.0$ ), with a subset of these findings corroborated via qRT-PCR (Fig. 2).

IncRNAs are gaining recognition as important functional components in eukaryotic gene regulation, with cis- and transregulatory mechanisms becoming more clearly characterized $(14,41,42)$. In general, lncRNAs can remodel the chromatin status of surrounding regions in a cis-fashion via co-transcription (competes for the transcription-binding complex between lncRNAs and their neighboring genes) by anchoring to RNA polymerase II or act as an artificial miRNA sponge to competitively inhibit the ability of miRNAs to bind their mRNA targets $(43,44)$. In addition, cis-regulatory lncRNAs may also regulate the transcription of nearby genes by facilitating access to enhancers and promoters for transcriptional machinery molecules (45). One classic cis-regulatory example 
involving two recently discovered lncRNAs, HOTTIP and HOTAIRM1, located at opposite ends of the HoxA cluster and help to promote the expression of neighboring Hox A genes (46).

Unlike cis-regulatory IncRNAs that act locally on the genomic region from which they are transcribed, transregulatory lncRNAs exert their effects by regulating the expression of target genes at geographically distant locations, even including different chromosomes $(42,46)$. For example, HOTAIR is a $2.2 \mathrm{~kb}$ lncRNA that originates from the HOXC locus, but recruits Polycomb Repressive Complex 2 (PRC2) to silence the HOXD locus of a different chromosome (42). For the trans-acting analysis, possible target genes of differentially expressed lncRNAs were identified using the RNAplex program which finds the optimal target sites of a query RNA relative to an mRNA target by computing secondary structures for their hybridization. The ability of RNAplex to afford a high degree of run-time efficiency without noticeable loss of specificity by performing a comparative target search allows poorly conserved interactions to be discarded. Subsequently, RNAplex is a powerful tool for the rapid and reliable prediction of RNA-RNA interactions, which is well suited for finding high-confidence ncRNA targets amongst a large genomic dataset $(27,28)$.

To increase the accuracy of target prediction, differentially expressed mRNA data were integrated with predicted lncRNA targets. To understand the potential functional roles of IncRNAs, GO category and KEGG pathway annotation were utilized to analyze the target gene pool. KEGG annotation showed the predicted target genes to be significantly enriched in 7 different pathways in GC tissue compared with matched normal gastric tissue. Among these pathways, the p53 signaling pathway (Fig. 4) was the most significant and has been previously implicated in GC pathogenesis $(29,30)$. Furthermore, construction of the IncRNA-mRNA correlation network displaying differentially expressed lncRNAs and their target genes (Fig. 5) implicated three target genes ATM, RRM2 and SERPINB5 which have been previously implicated in GC development and found to have involvement in p53 signaling in the present study (34-36). These findings indicate a possible role of the p53 signaling pathway in the dysregulation of lncRNAs during GC pathogenesis. Moreover, we postulate that the 10 differentially expressed lncRNAs (AK001094, AK057054, BC042436, uc003iqu.1, AK022971, uc003tfx.1, AK091139, AK001094, uc003dwf.3 and AF086216) and their predicted targets (ATM, RRM2 and SERPINB5) relate to the p53 signaling pathway and may play a significant collective role in $\mathrm{GC}$ pathogenesis.

In conclusion, this is the first study that describes the global expression profiling of lncRNAs and mRNAs relating to GC using microarray technology. In this study, we observed a large number of aberrantly expressed lncRNAs and mRNAs in GC samples when compared matched normal samples. Bioinformatic analysis to include lncRNA target prediction, GO category classification and KEGG pathway annotation enabled the uncovering of possible associations between lncRNAs and protein-coding genes to reveal potential functional roles of lncRNAs in GC pathogenesis. While the regulatory roles of several lncRNAs related to p53 signaling, the exact regulatory mechanisms still require further elucida- tion. Additionally, it is worth noting that each lncRNA-mRNA target pair serves as a strong candidate for GC diagnosis and therapeutics warranting further investigation.

\section{Acknowledgements}

This study was supported by the Science and Technology Planning Project of Shenzhen (201102176). We are deeply grateful to all donors who participated in this program.

\section{References}

1. Thiel A and Ristimäki A: Gastric cancer: basic aspects. Helicobacter 1: 26-29, 2012.

2. Leja M, Wex T and Malfertheiner P: Markers for gastric cancer premalignant lesions: where do we go? Digest Dis 30: 268-276, 2012

3. Guggenheim DE and Shah MA: Gastric cancer epidemiology and risk factors. J Surg Oncol 107: 230-236, 2013.

4. Jemal A, Bray F, Center MM, et al: Global cancer statistics. CA Cancer J Clin 61: 69-90, 2011.

5. Wagner AD, Grothe $\mathrm{W}$, Haerting J, et al: Chemotherapy in advanced gastric cancer: a systematic review and meta-analysis based on aggregate data. J Clin Oncol 24: 2903-2909, 2006.

6. Wang J, Wang Q, Liu H, et al: MicroRNA expression and its implication for the diagnosis and therapeutic strategies of gastric cancer. Cancer Lett 297: 137-143, 2010.

7. Gibb EA, Brown CJ and Lam WL: The functional role of long non-coding RNA in human carcinomas. Mol Cancer 10: 38-55, 2011.

8. Gutschner T and Diederichs S: The hallmarks of cancer: a long non-coding RNA point of view. RNA Biol 9: 703-719, 2012.

9. Griffiths JS, Saini HK, van Dongen S, et al: miRBase: tools for microRNA genomics. Nucleic Acids Res: 36: D154-D158, 2008.

10. Storz G, Opdyke JA and Zhang A: Controlling mRNA stability and translation with small, noncoding RNAs. Curr Opin Microbiol 7: 140-144, 2004.

11. Morey C and Avner P: Employment opportunities for non-coding RNAs. FEBS Lett 567: 27-34, 2004.

12. Liao Q, Liu C, Yuan X, et al: Large-scale prediction of long non-coding RNA functions in a coding-non-coding gene co-expression network. Nucleic Acids Res 39: 3864-3878, 2011.

13. Au PC, Zhu QH, Dennis ES, et al: Long non-coding RNA-mediated mechanisms independent of the RNAi pathway in animals and plants. RNA Biol 8: 404-414, 2011.

14. Ma H, Hao Y, Dong X, et al: Molecular mechanisms and function prediction of long noncoding RNA. Sci World J 2012: 541786, 2012.

15. Sui W, Yan Q, Li H, et al: Genome-wide analysis of long noncoding RNA expression in peripheral blood mononuclear cells of uremia patients. J Nephrol 26: 731-738, 2013.

16. Yin Z, Guan D, Fan Q, et al: lncRNA expression signatures in response to enterovirus 71 infection. Biochem Biophys Res Commun 430: 629-633, 2013.

17. Yu G, Yao W, Wang J, et al: LncRNAs expression signatures of renal clear cell carcinoma revealed by microarray. PLoS One 7: e42377, 2012.

18. Chen G, Wang Z, Wang D, et al: LncRNADisease: a database for long-non-coding RNA-associated diseases. Nucleic Acids Res 41: D983-D986, 2013.

19. Harries LW: Long non-coding RNAs and human disease. Biochem Soc 40: 902-906, 2012.

20. Cao WJ, Wu HL, He BS, et al: Analysis of long non-coding RNA expression profiles in gastric cancer. World J Gastroenterol 19: 3658-3664, 2013.

21. Pfaffl MW: A new mathematical model for relative quantification in real-time RT-PCR. Nucleic Acids Res 29: 45, 2001.

22. Sui W, Lin H, Peng W, et al: Molecular dysfunctions in acute rejection after renal transplantation revealed by integrated analysis of transcription factor, microRNA and long noncoding RNA. Genomics 102: 310-322, 2013.

23. Szafranski P, Dharmadhikari AV, Brosens E, et al: Small noncoding differentially methylated copy-number variants, including lncRNA genes, cause a lethal lung developmental disorder. Genome Res 23: 23-33, 2013. 
24. Jia H, Osak M, Bogu GK, et al: Genome-wide computational identification and manual annotation of human long noncoding RNA genes. RNA 16: 1478-1487, 2010.

25. Han L, Zhang K, Shi Z, et al: LncRNA profile of glioblastoma reveals the potential role of lncRNAs in contributing to glioblastoma pathogenesis. Int J Oncol 40: 2004-2012, 2012.

26. Bu Q, Hu Z, Chen F, et al: Transcriptome analysis of long noncoding RNAs of the nucleus accumbens in cocaine-conditioned mice. J Neurochem 123: 790-799, 2012.

27. Tafer $\mathrm{H}$ and Hofacker IL: RNAplex: a fast tool for RNA-RNA interaction search. Bioinformatics 24: 2657-2663, 2008.

28. Tafer H, Amman F, Eggenhofer F, et al: Fast accessibilitybased prediction of RNA-RNA interactions. Bioinformatics 27 1934-1940, 2011

29. Ryu DS, Lee HS, Lee GS, et al: Effects of the ethylacetate extract of Orostachys japonicus on induction of apoptosis through the p53-mediated signaling pathway in human gastric cancer cells. Biol Pharm Bull 35: 660-665, 2012.

30. Liu J, Xie YS, Wang FL, et al: Cytotoxicity of 5-Aza-2'deoxycytidine against gastric cancer involves DNA damage in an ATM-P53 dependent signaling pathway and demethylation of P16(INK4A). Biomed Pharmacother 67: 78-87, 2013.

31. Ma GF, Chen SY, Sun ZR, et al: FoxP3 inhibits proliferation and induces apoptosis of gastric cancer cells by activating the apoptotic signaling pathway. Biochem Biophys Res Commun 430: 804-809, 2013.

32. Yu RX, Hu XM, Xu SQ, et al: Effects of fucoxanthin on proliferation and apoptosis in human gastric adenocarcinoma MGC-803 cells via JAK/STAT signal pathway. Eur J Pharmacol 657: 10-19, 2011.

33. To KF, Chan MW, Leung WK, et al: Constitutional activation of IL-6-mediated JAK/STAT pathway through hypermethylation of SOCS-1 in human gastric cancer cell line. Br J Cancer 91: $1335-1341,2004$

34. Kim JW, Im SA, Kim MA, et al: Ataxia-telangiectasia mutated (ATM) protein expression with microsatellite instability in gastric cancer as prognostic marker. Int J Cancer 134: 72-80, 2013.
35. Morikawa T, Hino R, Uozaki H, et al: Expression of ribonucleotide reductase M2 subunit in gastric cancer and effects of RRM2 inhibition in vitro. Hum Pathol 41: 1742-1748, 2010.

36. Terashima M, Maesawa C, Oyama K, et al: Gene expression profiles in human gastric cancer: expression of maspin correlates with lymph node metastasis. Br J Cancer 92: 1130-1136, 2005.

37. Hudler P: Genetic aspects of gastric cancer instability. Sci World J 2012: 761909, 2012.

38. Qiu MT, Hu JW, Yin R, et al: Long noncoding RNA: an emerging paradigm of cancer research. Tumour Biol 34: 613-620, 2013.

39. Qi P and Du X: The long non-coding RNAs, a new cancer diagnostic and therapeutic gold mine. Mod Pathol 26: 155-165, 2013.

40. Yang F, Bi J, Xue X, et al: Up-regulated long non-coding RNA H19 contributes to proliferation of gastric cancer cells. FEBS J 279: 3159-3165, 2012.

41. Kim ED and Sung S: Long noncoding RNA: unveiling hidden layer of gene regulatory networks. Trends Plant Sci 17: 16-21, 2012.

42. De LF and Dean C: Long non-coding RNAs and chromatin regulation. Curr Opin Plant Biol 14: 168-173, 2011.

43. Qu Z and Adelson DL: Identification and comparative analysis of ncRNAs in human, mouse and zebrafish indicate a conserved role in regulation of genes expressed in brain. PLoS One 7: e52275, 2012

44. Wilusz JE, Sunwoo H and Spector DL: Long noncoding RNAs: functional surprises from the RNA world. Genes Dev 23: 1494-1504, 2009.

45. Ponjavic J, Oliver PL, Lunter G, et al: Genomic and transcriptional co-localization of protein-coding and long non-coding RNA pairs in the developing brain. PLoS Genet 5: e1000617, 2009.

46. Prensner JR and Chinnaiyan AM: The emergence of lncRNAs in cancer biology. Cancer Discov 1: 391-407, 2011. 